\title{
Intraoperative Goal-Directed Therapies in Femoral and Pelvic Osteotomies in Children and In-Hospital Postoperative Outcomes
}

\author{
Claudine Kumba1 ${ }^{(0)}$, Mathilde Gaume², Arayik Barbarian1, Zaga Péjin² \\ ${ }^{1}$ Department of Pediatric Anesthesia and Critical Care, Hôpital Universitaire Necker Enfants Malades, Assistance Publique \\ Hôpitaux de Paris, APHP, Université de Paris, Paris, France \\ ${ }^{2}$ Department of Pediatric Orthopedic Surgery, Hôpital Universitaire Necker Enfants Malades, Assistance Publique, Hôpitaux de \\ Paris, APHP, Université de Paris, Paris, France \\ Email: claudine.kumba@aphp.fr, mathilde.gaume@aphp.fr, arayik.barbarian@aphp.fr, zagorka.pejin@aphp.fr
}

How to cite this paper: Kumba, C., Gaume, M., Barbarian, A. and Péjin, Z. (2021) Intraoperative Goal-Directed Therapies in Femoral and Pelvic Osteotomies in Children and In-Hospital Postoperative Outcomes. Open Journal of Orthopedics, 11, 327-334.

https://doi.org/10.4236/ojo.2021.1111031

Received: October 8, 2021

Accepted: November 15, 2021

Published: November 18, 2021

Copyright $\odot 2021$ by author(s) and Scientific Research Publishing Inc. This work is licensed under the Creative Commons Attribution International License (CC BY 4.0).

http://creativecommons.org/licenses/by/4.0/

\section{(c) (i) Open Access}

\begin{abstract}
Background: Femoral and pelvic osteotomies are potential hemorrhagic interventions where transfusion requirements can be necessary. Objective: We undertook a secondary analysis of patients who underwent femoral and pelvic osteotomy in the initial cohort. The objective of this secondary analysis was to describe intraoperative and postoperative outcomes and to describe intraoperative management in these patients in terms of blood product management and fluid and hemodynamic therapy with the aim of implementing optimization management protocols for postoperative outcome improvement. Methods: A secondary analysis of patients who underwent femoral and pelvic osteotomy surgery was included in the initial retrospective study. Results: There were eighteen patients with a mean age of $104 \pm 47.1$ months. Four (22.2\%) patients had intraoperative and/or postoperative complications. One patient (5.6\%) had an intraoperative hemorrhagic shock, two patients (11.1\%) had postoperative neurologic failure, and one patient (5.6\%) had postoperative wound sepsis. The transfusion rate was $50 \%$ in nine patients. Conclusion: Femoral and pelvic osteotomies are interventions where blood, transfusion and fluid requirements can be increased; thus, this implies the necessity of a global patient blood management protocol with point-of-care tests and fluid- and hemodynamic-guided protocols with validated tools in children for intraoperative and postoperative outcome optimization.
\end{abstract}

\section{Keywords}

Femoral Osteotomy, Pelvic Osteotomy, Patient Blood Management, Fluid and Hemodynamic Goal-Directed Therapy, Children, Postoperative Outcome 


\section{Introduction}

Femoral and pelvic osteotomies are common interventions in children with cerebral palsy [1] [2]. This functional surgery aims to improve the quality of life in fragile patients in terms of pain and postural discomfort that can be observed in hip displacement and can impact the daily quality of life in terms of positioning and nursing [3] [4]. Femoral and pelvic osteotomies are also performed in patients who have sequelae of developmental dysplasia of the hip, sequelae of septic arthritis of the hip, Legg-Calvé-Perthes disease, skeletal dysplasia, etc. [5] [6] [7].

Femoral and pelvic osteotomies are potential hemorrhagic interventions where transfusion requirements can be necessary due to the surgical characteristics of the interventions. A retrospective observational study was undertaken to determine predictors of adverse intraoperative and postoperative outcomes in patients undergoing neurosurgery and abdominal and orthopedic surgery at our hospital Necker Enfants Malades University Hospital [8]. Age, American Society of Anesthesiologists Score (ASA), emergency situations, surgery and transfusion were independent predictors of intraoperative and postoperative outcomes in terms of morbidity, reoperations, length of stay in the intensive care unit (LOSICU), length of stay in hospital in the conventional hospitalization ward (LOS), total length of stay in hospital (LOSICU + LOS) and length of mechanical ventilation (LMV) [8] [9]. We undertook a secondary analysis of patients who underwent femoral and pelvic osteotomy in the initial cohort. Similar secondary analyses have been undertaken in other surgical interventions [10] [11] [12]. The objective of this secondary analysis was to describe intraoperative and postoperative outcomes and to describe intraoperative management in these patients in terms of blood product management and fluid and hemodynamic therapy with the aim of implementing optimization management protocols for postoperative outcome improvement.

\section{Methods and Materials}

Methods: Secondary analysis of patients who underwent femoral and pelvic osteotomy surgery was included in the initial retrospective study [8].

The study was approved by the Ethics Committee of Necker Enfants Malades University Hospital under registration number 2017-CK-5-R1 on 21 March 2017.

Inclusion: Patients were retrospectively included from 1 January 2014 to 17 May 2017.

Since we aimed to include pediatric patients, the inclusion criteria were patients aged less than 18 years who underwent femoral and pelvic osteotomy included in the initial retrospective study.

The exclusion criteria were patients aged more than 18 years old and patients who did not undergo femoral or pelvic osteotomy included in the initial study.

Intraoperative and postoperative patient management: In our hospital, patients who underwent femoral and pelvic osteotomy were managed intraopera- 
tively according to a defined protocol as described hereafter.

Preoperatively, patients had iron supplementation at $5-10 \mathrm{mg} / \mathrm{kg} / \mathrm{day}$, and a complete blood cell count and blood group were available.

Induction of anesthesia was inhalational with sevoflurane in an oxgen-air mixture and sufentanil as an IV bolus of $0.2 \mu \mathrm{g} / \mathrm{kg}$. Two large-bore peripheral intravenous (IV) lines were inserted. Airway was secured with oro-tracheal intubation. All patients had nasogastric tubing, an indwelling bladder catheter inserted, a central core temperature probe, a Bair Hugger ${ }^{\circledast}$ and an IV fluid warming device.

Maintenance of anesthesia was performed with sevoflurane. An epidural lumbar catheter for intraoperative and postoperative analgesia was inserted after the induction of anesthesia. Intraoperative epidural analgesia was performed with levobupivacaine $0.0625 \%$ as an infusion rate of $0.2-0.3 \mathrm{~mL} / \mathrm{kg} / \mathrm{h}$.

Antibiotic prophylaxis was performed with a $50 \mathrm{mg} / \mathrm{kg}$ IV bolus of cefazolin. Tranexamic acid was administered as a $30 \mathrm{mg} / \mathrm{kg}$ IV bolus over 30 minutes followed by an intravenous infusion of $10 \mathrm{mg} / \mathrm{kg} / \mathrm{h}$.

Fluid therapy was performed with crystalloids as a $10-20 \mathrm{~mL} / \mathrm{kg}$ bolus (Ringer Lactate $^{\circledast}$ or chloride sodium $0.9 \%$ ) or colloids as a $30 \mathrm{~mL} / \mathrm{kg}$ bolus (plasmion ${ }^{\circledast}$ or voluven $^{\circledast}$ ). Intraoperative hemoglobin levels were monitored on a regular basis.

Postoperative analgesia was performed with epidural analgesia with levobupivacaine $0.0625 \%$ at an infusion rate of $0.1-0.2 \mathrm{~mL} / \mathrm{kg} / \mathrm{h}, \mathrm{IV}$ acetaminophen at $15 \mathrm{mg} / \mathrm{kg} / 6 \mathrm{~h}$, IV ketoprofen at $1 \mathrm{mg} / \mathrm{kg} / 12 \mathrm{~h}$ or oral ibuprofen at $10 \mathrm{mg} / \mathrm{kg} / 8 \mathrm{~h}$ and IV clonidine at $1-2 \mu \mathrm{g} / \mathrm{kg}$. A rescue postoperative analgesia protocol was performed with IV nalbuphine at $0.2 \mathrm{mg} / \mathrm{kg} / 6 \mathrm{~h}$ or with oral morphine at 1 $\mathrm{mg} / \mathrm{kg} /$ day divided 4 - 6 times.

Postoperative spasticity prevention and treatment were realized with oral or intrarectal diazepam at $50 \mu \mathrm{g} / \mathrm{kg} / 4 \mathrm{~h}$.

Depending on general status and comorbidity, patients were transferred postoperatively in the pediatric intensive care unit (PICU) for surveillance or in the postinterventional care unit (PACU) and afterward in the conventional surgical hospitalization ward.

Intravenous iron supplementation at postoperative day 1 was performed depending on postoperative hemoglobin levels.

Statistics: Statistics were analyzed with XLSTAT 2020.4.1. software. Continuous variables were expressed as medians with ranges or means with standard deviations. Category variables were described in proportions.

\section{Results}

Table 1 illustrates the general characteristics.

There were eighteen patients with a mean age of $104 \pm 47.1$ months and a median weight of 22 [16 - 55] kilograms. Eleven (61.1\%), four (22.2\%), and three (16.7\%) patients had ASA scores of II, III and IV, respectively. Eleven (61.1\%) and seventeen (38.9\%) patients underwent femoral and pelvic osteotomy, respectively. There were seventeen (94.4\%) elective interventions and one (5.6\%) urgent surgery. 
Table 1. General characteristics.

\begin{tabular}{|c|c|}
\hline Characteristic & $\mathrm{N}=18$ \\
\hline Mean age \pm standard deviation in months & $104 \pm 47.1$ \\
\hline Median weight [interquartile range] in kilograms & $22[16-55]$ \\
\hline ASA II n (\%) & $11(61.1)$ \\
\hline ASA III n (\%) & $4(22.2)$ \\
\hline ASA IV n (\%) & $3(16.7)$ \\
\hline Femoral osteotomy n (\%) & $11(61.1)$ \\
\hline Pelvic osteotomy n (\%) & $7(38.9)$ \\
\hline Emergency surgery n (\%) & $1(5.6)$ \\
\hline Elective surgery n (\%) & $17(94.4)$ \\
\hline Re-operation n (\%) & $0(0)$ \\
\hline $\begin{array}{l}\text { Patients with intra-operative and or postoperative complications } \\
\text { (organ failure or sepsis) } \mathrm{n}(\%)\end{array}$ & $4(22.2)$ \\
\hline Intra-operative hemorrhagic shock n (\%) & $1(5.6)$ \\
\hline Postoperative neurologic failure $\mathrm{n}(\%)$ & $2(11.1)$ \\
\hline Postoperative surgical wound sepsis n (\%) & $1(5.6)$ \\
\hline In-hospital mortality n (\%) & $0(0)$ \\
\hline Transfusion $\mathrm{n}(\%)$ & $9(50)$ \\
\hline Median length of intensive care unit stay in days [range] & $0[0-12]$ \\
\hline Median length of hospital stay in days [range] & $6[1-23]$ \\
\hline Median total length of hospital stay in days [range] & $6[2-32]$ \\
\hline Median length of mechanical ventilation in days [range] & $0[0-2]$ \\
\hline Median packed red blood cell volume in units [range] & $0[0-2]$ \\
\hline Median fresh frozen plasma volume in units [range] & $0[0-0]$ \\
\hline Median concentrated platelet units [range] & $0[0-0]$ \\
\hline Mean preoperative hemoglobin levels \pm standard deviation in $\mathrm{g} / \mathrm{dL}$ & $10.36 \pm 1.7$ \\
\hline Mean postoperative hemoglobin levels \pm standard deviation in $\mathrm{g} / \mathrm{dL}$ & $10.3 \pm 1.0$ \\
\hline Median crystalloid volume in $\mathrm{mL}$ [range] & $1150[750-2000]$ \\
\hline Median colloid volume in $\mathrm{mL}$ [range] & $400[0-900]$ \\
\hline
\end{tabular}

Four (22.2\%) patients had intraoperative and/or postoperative complications. One patient (5.6\%) had intraoperative hemorrhagic shock, two patients (11.1\%) had postoperative neurologic failure, and one patient (5.6\%) had postoperative wound sepsis. There were no reoperations and no in-hospital mortality. Nine patients (50\%) had intraoperative transfusion with packed red blood cell units (PRBCs). The median length of intensive care unit stay (LOSICU) was 0 [0 - 12] 
days. The median length of hospital stay (LOS) in the conventional surgical ward was 6 [ 1 - 23] days. The median total length of hospital stay (TLOS $=$ LOSICU + LOS) was 6 [2 - 32] days. The median length of mechanical ventilation was 0 [0 $2]$ days. The median packed red blood cell volume was 0 [0 - 2] units. No fresh frozen plasma or platelets were administered. The mean preoperative hemoglobin level was $10.36 \pm 1.7 \mathrm{~g} / \mathrm{dL}$. The mean postoperative hemoglobin level was $10.3 \pm 1.0 \mathrm{~g} / \mathrm{dL}$. The median crystalloid volume was $1150[750-2000] \mathrm{mL}$, and the median colloid volume was 400 [0 - 900] $\mathrm{mL}$.

Table 2 illustrates co-morbidities.

The most common comorbidities were sequelae of hip arthritis, developmental dysplasia of the hip, and Legg-Calvé-Perthes disease in nine patients (50\%), followed by cerebral palsy in 3 patients (16.7\%), among which two $(11.1 \%)$ had cerebral anoxic lesions and one (5.6\%) had psychomotor deficiency. Two patients (11.1\%) had Ewing's sarcoma and Gorlin's syndrome. One patient (5.6\%) had osteogenesis imperfecta.

\section{Discussion}

In this secondary analysis, the proportion of patients with complications and transfusion was $22 \%$ and $50 \%$ respectively. Femoral and pelvic osteotomies are interventions performed in patients who can have a fragile general status, as illustrated by the comorbidities in our cohort. In these surgical settings, blood loss and fluid requirements can be high, as illustrated by the transfusion rate and the amount of fluid administered intraoperatively. This implies the inclusion of transfusion protocols guided with point-of-care tests and fluid and hemodynamic therapy protocols for optimal intraoperative patient blood management and fluid therapy in this surgical setting.

As described in previous studies, these goal-directed therapies have the objectives of improving postoperative outcomes [10]-[19]. Transfusion and intraoperative nonoptimal hemodynamic parameters have been associated with adverse postoperative outcomes in surgical patients [8] [9] [19]. Non-optimal preoperative, intraoperative and postoperative hemoglobin levels have been

Table 2. Co-morbidities.

\begin{tabular}{cc}
\hline Co-morbidity & Number of patients (\%) \\
\hline $\begin{array}{c}\text { Sequelae of hip arthritis, developmental } \\
\text { dysplasia of the hip, Legg-Calvé-Perthes disease } \\
\text { Cerebral anoxic lesions }\end{array}$ & $9(50)$ \\
Ewing's sarcoma & $2(11.1)$ \\
Gorlin's syndrome & $2(11.1)$ \\
Osteogenesis imperfecta & $2(11.1)$ \\
Psychomotor deficiency & $1(5.6)$ \\
\hline
\end{tabular}


correlated with adverse postoperative outcomes in surgical patients [20]. Optimal hemoglobin levels depend on patient status, comorbidities and surgical context. Optimal hemoglobin levels are those that do not favor the development of a dependent relationship between oxygen consumption and oxygen delivery [16] [17]. Anemia has been reported to be correlated with in-hospital adverse outcomes [21] [22]. Benefits and risks related to transfusion and anemia need to be balanced when making a decision for blood transfusion.

Fluid therapy with aortic blood flow peak velocity variation with transthoracic echocardiography or esophageal Doppler probe is a validated tool in children to assess fluid responsiveness in patients under anesthesia [18] [23].

This secondary analysis of patients who underwent femoral and pelvic osteotomies was an occasion to introduce and encourage the integration of goal-directed therapies in intraoperative patient management to improve and upgrade postoperative evolution in these fragile patients.

\section{Conclusion}

Femoral and pelvic osteotomies are interventions where blood, transfusion and fluid requirements can be increased; thus, this implies the necessity of global patient blood management protocols with point-of-care tests and fluid- and hemodynamic-guided protocols with validated tools in children for intraoperative and postoperative outcome optimization.

\section{Conflicts of Interest}

The authors declared no conflicts of interest.

\section{Authors Contributions}

Claudine Kumba conceptualized and designed the study and drafted the initial manuscript. She designed the data collection instruments, collected data, carried out initial and final analyses.

Zaga Péjin reviewed the manuscript.

Mathilde Gaume reviewed the manuscript.

Arayik Barbarian reviewed the manuscript.

\section{Presentation of Preliminary Results}

This manuscript was registered as a preprint under the DOI registration number https://doi.org/10.21203/rs.3.rs-777279/v2 on Research Square a preprint platform.

\section{Ethics Approval}

This study received approval from the Ethics Committee of Necker on 21 March 2017 under registration number 2017-CK-5-R1 and waived patient consent.

\section{References}

[1] Alassaf, N., Saran, N., Benaroch, T. and Hamdy, R.C. (2018) Combined Pelvic and 
Femoral Reconstruction in Children with Cerebral Palsy. Journal of International Medical Research, 46, 475-484. https://doi.org/10.1177/0300060517723797

[2] Min, J.J., Kwon, S.S., Sung, K.H., Lee, K.M., Chung, C.Y. and Park, M.S. (2021) Remodeling of Femoral Head Deformity after Hip Reconstructive Surgery in Patients with Cerebral Palsy. Bone \& Joint Journal, 103-B, 198-203. https://doi.org/10.1302/0301-620X.103B1.BJJ-2020-1339.R1

[3] Sung, K.H., Kwon, S.S., Chung, C.Y., Lee, K.M., Kim, J., Lee, S.Y., et al. (2018) Fate of Stable Hips after Prophylactic Femoral Varization Osteotomy in Patients with Cerebral Palsy. BMC Musculoskeletal Disorders, 19, Article No. 130. https://doi.org/10.1186/s12891-018-2049-Z

[4] Terjesen, T. (2019) Femoral and Pelvic Osteotomies for Sever Hip Displacement in Non-Ambulatory Children with Cerebral Palsy: A Prospective Population-Based Study of 31 Patients with 7 Years Follow-up. Acta Orthopaedica, 90, 614-621. https://doi.org/10.1080/17453674.2019.1675928

[5] Andrzejewski, A., Péjin, Z., Finidori, G., Badina, A., Glorion, C. and Wicart, P. (2021) Can Chiari Osteotomy Favorably Influence Long-Term Hip Degradation in Multiple Epiphyseal Dysplasia and Pseudoachondroplasia? Journal of Pediatric Orthopaedics, 41, e135-e140. https://doi.org/10.1097/BPO.0000000000001708

[6] Bhatti, A., Kumar, J. and Butt, S.A. (2014) Outcome of One-Stage Combined Open Reduction, Pelvic and Derotation Femoral Osteotomy in Congenital Dislocated Hips of Children Younger than Three Years of Age. Journal of Pakistan Medical Association, 64, 1015-1020.

[7] Rosello, O., Solla, O.I., Chau, E., El Hayek, T., Clement, J.L. and Rampal, V. (2018) Advanced Containment Methods for Legg-Calvé-Perthes Disease: Triple Pelvic Osteotomy versus Chiari Osteotomy. HIP International, 28, 297-301. https://doi.org/10.5301/hipint.5000569

[8] Kumba, C., Cresci, F., Picard, C., Thiry, C., Albinni, S. and Orliaguet, G. (2017) Transfusion and Morbi-Mortality Factors: An Observational Descriptive Retrospective Pediatric Cohort Study. Journal of Anesthesia \& Critical Care: Open Access, 8 , Article No. 00315. https://doi.org/10.15406/jaccoa.2017.08.00315

[9] Kumba, C., Lenoire, A., Cairet, P., Dogaru-Dedieu, E., Belloni, I. and Orliaguet, G. (2018) Is Transfusion an Independent Risk Factor of Postoperative Outcome in Pediatric Orthopedic Surgical Patients? A Retrospective Study. Journal of Emergency Medicine \& Critical Care, 4, Article No. 7. https://doi.org/10.13188/2469-4045.1000018

[10] Kumba, C. (2022) Liver Transplantation in Children and Impact of Intraoperative Goal-Directed Therapies on Postoperative Outcome. SOJ Pediatrics and Clinical Neonatology, 2, 1-7. (To be published on 28 January 2022)

https://doi.org/10.53902/SOJPCN.2022.02.000512

[11] Kumba, C. and Miladi, L. (2021) Scoliosis in Children: Impact of Goal-Directed Therapies on Intraoperative and Postoperative Outcomes. Open Journal of Orthopedics, 11, 315-326. https://doi.org/10.4236/ojo.2021.1110030

[12] Kumba, C. (2021) Patient Blood Management in Craniosynostosis Surgery. Open Journal of Modern Neurosurgery, 11, 211-222.

https://doi.org/10.4236/ojmn.2021.114025

[13] Kumba, C. and Tréluyer, J. (2020) Perspectives and Evolution of Intraoperative Transfusion Goal Directed Protocols with Viscoelastic Methods and Perioperative Outcomes in Children. Research in Pediatrics \& Neonatology, 4, Article ID: RPN.000582. https://doi.org/10.31031/RPN.2020.04.000582 
[14] Kumba, C., Querciagrossa, S., Harte, C., Willems, A., De Cock, A., Blanc, T., et al. (2019) A Systematic Review and Meta-Analysis of Goal Directed Intra-Operative Transfusion Protocols Guided by Viscoelastic Methods and Perioperative Outcomes in Children. International Journal of Recent Scientific Research, 10, 31466-31471.

[15] Haas, T., Spielmann, N., Restin, T., Schmidt, A.R., Schmugge, M. and Cushing, M.M. (2016) Economic Aspects of Intraoperative Coagulation Management Targeting Higher Fibrinogen Concentrations during Major Craniosynostosis Surgery. Pediatric Anesthesia, 26, 77-83. https://doi.org/10.1111/pan.12784

[16] Kumba, C. (2020) Physiology Principles Underlying Goal Directed Therapies in Children. Research in Pediatrics \& Neonatology, 4, Article ID: RPN.000591. https://doi.org/10.31031/RPN.2020.04.000591

[17] Kumba, C. (2020) Rationale of Goal Directed Therapies in Children. Advances in Pediatric Research, 7, Article No. 42.

https://www.longdom.org/articles/rationale-of-goal-directed-therapies-in-c hildren.pdf

[18] Kumba, C. (2020) Goal Directed Fluid and Hemodynamic Therapy and Postoperative Outcomes in Children: Value of Transthoracic Echocardiographic Aortic Blood Flow Peak Velocity Variation: A Multi-Centre Randomized Controlled Trial Protocol. Advances in Pediatric Research, 7, Article No. 35. https://doi.org/10.35248/2385-4529.20.7.35

[19] Kumba, C., Willems, A., Querciagrossa, S., Harte, C., Blanc, T., De Cock, A., et al. (2019) A Systematic Review and Meta-Analysis of Intraoperative Goal Directed Fluid and Haemodynamic Therapy in Children and Postoperative Outcome. Journal of Emergency Medicine \& Critical Care, 5, 1-9. https://doi.org/10.13188/2469-4045.1000020

[20] Kumba, C. (2021) Hemoglobin Levels and Postoperative Outcome in Pediatric Surgical Patients. Research Square. (Preprint) https://doi.org/10.21203/rs.3.rs-669069/v1

[21] Faraoni, D. and Goobie, S. (2016) Relationship between Preoperative Anemia and In-Hospital Mortality in Children Undergoing Noncardiac Surgery. Anesthesia \& Analgesia, 123, 1582-1587. https://doi.org/10.1213/ANE.0000000000001499

[22] Kumba, C. (2019) Iron Deficiency with or without Anemia and Perspectives of Perioperative Management in Children. Advances in Pediatric Research, 6, Article No. 33. https://doi.org/10.35248/2385-4529.19.6.33

[23] Gan, H., Cannesson, M., Chandler, J.R. and Ansermino, J.M. (2013) Predicting Fluid Responsiveness in Children: A Systematic Review. Anesthesia \& Analgesia, 117, 1380-1392. https://doi.org/10.1213/ANE.0b013e3182a9557e 